\title{
TeV emission and jet formation in M87
}

\section{Frank M. Rieger}

Max-Planck-Institut für Kernphysik, 69117 Heidelberg, Germany

E-mail: frank.rieger@mpi-hd.mpg.de

\section{Amir Levinson}

School of Physics and Astronomy, Tel Aviv University, Tel Aviv 69978, Israel

E-mail: levinsonewise.tau.ac.il

\begin{abstract}
The detection of rapidly variable $\mathrm{TeV}$ emission on timescales of $\sim 1$ day in M87 implies a source size of a few Schwarzschild radii $r_{s}$, and suggests that the $\mathrm{TeV}$ emission may originate from unscreened electric fields in the black hole magnetospheres. The presence of the VLBA jet, on the other hand, indicates that a force-free (ideal) MHD flow is established on scales $<50 r_{s}$. We show that inverse Compton scattering of ambient photons by electrons accelerated in the gap can initiate a pair cascade above the gap, that results in a sufficiently large multiplicity to ensure a force-free flow. Photons having energies below a few TeV can escape freely. The VHE power radiated by the gap can easily account for the luminosity of the $\mathrm{TeV}$ source detected by, e.g., H.E.S.S. Intermittencies of the cascade process will give rise to the observed variability of the $\mathrm{TeV}$ emission, and the resultant force-free outflow, as indicated by the morphological changes of the VLBA jet.
\end{abstract}

25th Texas Symposium on Relativistic Astrophysics

December 6-10, 2010

Heidelberg, Germany 


\section{Introduction}

Rotating, supermassive black holes (BHs) have often been considered as putative sources for the production of ultra-high energy (UHE) cosmic- and very high energy (VHE) gamma-rays, e.g., see $[1,2,3,4,5]$. With the recent detection of VHE $\gamma$-rays from the Virgo cluster galaxy M87 [6, 7, 8], such near-BH-scenarios have gained an additional impetus: While the rapid VHE flux variations, on timescales $t_{\mathrm{obs}} \sim(1-2)$ days, as observed by the H.E.S.S. telescope array in 2005 already suggested a small size $\left(r \sim t_{\text {obs }} c \sim\right.$ a few gravitational radii $)$ of the $\gamma$-ray emitting region in M87 [6], its proper location still remained ambiguous. In the meanwhile, additional high-resolution $\left(\sim 50 r_{s}\right)$ radio VLBA imaging has shown that the $\gamma$-ray flaring activity in M87 can be accompanied by an increase in the radio flux close to core, indicating that the required energetic charged particles are accelerated in the vicinity of the central black hole itself [9]. Further observations are currently needed to fully establish the conditions under which such a situation is obtained. From a theoretical point of view, these observations provide support for magnetospheric models, where the relevant non-thermal processes are considered to occur at the base of a rotating black-hole-jet magnetosphere [10, 11, 12].

\section{The central engine in M87}

The radio galaxy M87 is located at a distance of $d \sim 16 \mathrm{Mpc}$ and known to host one of the most massive $\mathrm{BH}, M_{\mathrm{BH}} \simeq(3-6) \times 10^{9} M_{\odot}[13,14]$. Given its large mass, M87 is highly underluminous, with an estimated bolometric luminosity of only $L_{b} \sim 10^{42} \mathrm{erg} / \mathrm{s}$ [15]. M87 has thus often been considered to be a prototype galaxy for the presence of a radiatively inefficient accretion flow (RIAF), e.g. [17, 18].

\subsection{RIAF conditions in M87}

Chandra X-ray observations of M87 give an upper limit on the Bondi accretion rate (in terms of the Eddington accretion rate) of $\dot{m}_{B} \simeq 1.6 \times 10^{-3}$ [16]. Because the gas in an RIAF has angular momentum, the radial inflow speed is smaller (compared to the Bondi case) by a factor $\sim \alpha$, where $\alpha \sim 0.1$ is the usual viscosity coefficient [19]. The anticipated RIAF accretion rate in M87 thus becomes $\dot{m} \sim \alpha \dot{m}_{B} \sim 10^{-4}$. With this, the equipartition magnetic field strength is

$$
B \simeq 4 \times 10^{2} \dot{m}_{-4}^{1 / 2} M_{9}^{-1 / 2}\left(r / r_{s}\right)^{-5 / 4} \quad \mathrm{G} .
$$

where $m_{-4}=\dot{m} / 10^{-4}$ and $M_{9}=M_{\mathrm{BH}} / 10^{9} M_{\odot}$. Provided the magnetic field is sufficiently organized, a spinning black hole threatened by it, can build up a rotating magnetosphere and thereby trigger the formation of a relativistic outflow. When the charge density in the magnetosphere is sufficiently small, regions of unscreened electric fields (i.e., gaps) form in which particles can get quickly accelerated to high energies. The situation can then resemble the one shown in Fig. 1.

\subsection{ADAF emission spectrum}

The classical RIAF prototype is the two-temperature, optically-thin advection-dominated accretion flow (ADAF) [20]. The released viscous energy is here assumed to go primarily into ion heating, 


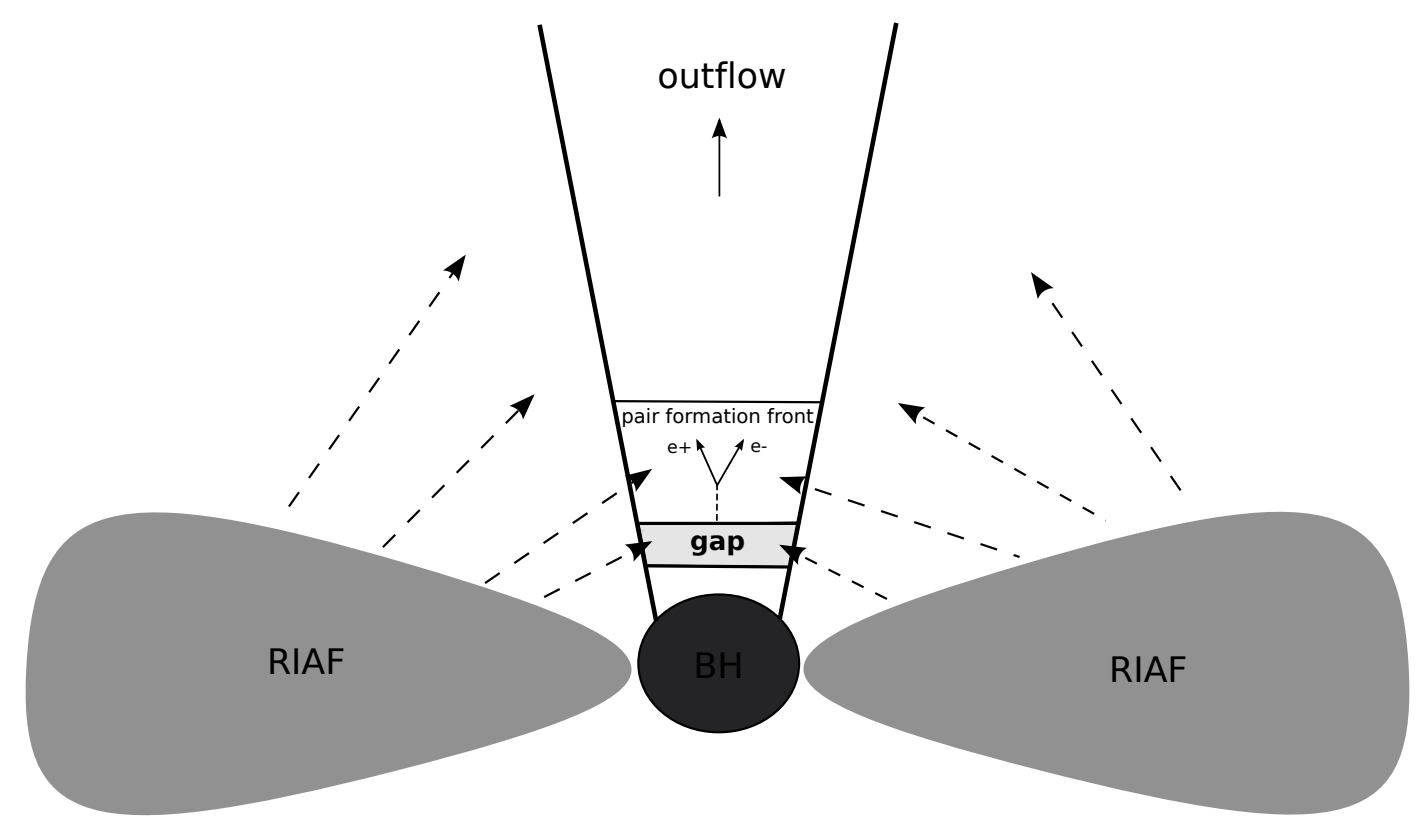

Figure 1: Schematic representation of the considered magnetospheric structure: A vacuum gap of height $h<r_{s}$ accelerates particles ( $e^{ \pm}$, injected by annihilation of RIAF MeV photons) to high Lorentz factors. The gap is exposed to soft radiation emitted from the surrounding disk (RIAF). Curvature emission and inverse Compton (IC) up-scattering produce VHE photons with a spectrum extending up to $10^{4} \mathrm{TeV}$. Photons having energies below a few $\mathrm{TeV}$ can escape freely to infinity. Interactions of IC photons, having energies well above $10 \mathrm{TeV}$, with the ambient radiation field initiate pair cascades just above the gap, leading to a large multiplicity. A force-free outflow is established above the pair formation front and appears as VLBA jet. Intermittencies of the cascade process, induced by modest changes in accretion rate, give rise to the variability of the $\mathrm{TeV}$ emission and to fluctuations of the resultant force-free outflow.

while cooling (e.g., synchrotron) is mainly by the electrons. The ion temperature is thus close to virial, $T_{p} \sim 3 \times 10^{12}\left(r_{g} / r\right) \mathrm{K}$, and the electron temperature at radii $r<10^{2} r_{s}$ is typically in the range $T_{e} \sim\left(10^{9}-10^{10}\right) \mathrm{K}$.

The ADAF broadband spectrum then ranges from radio to hard X-ray frequencies, see Fig. 2 for an illustration $[21,22]$. The radio to sub-mm regime is related to the synchrotron emission of the relativistic thermal electrons. The emission at the highest peak frequency $v_{p} \sim 10^{12} \mathrm{~Hz}$ (for the considered $\dot{m}$ ) comes from the innermost part of the flow. Compton upscattering of the low-energy synchrotron photons by the ADAF electrons leads to a hard power law tail extending up to energies $h v \sim k T_{e}$. Compton scattering becomes less important with decreasing accretion rates, and the spectrum becomes steeper (softer). For sufficiently low $\dot{m}$, distinct Compton peaks can appear. This is related to the increase in electron temperature with decreasing accretion rates.

For low $\dot{m}$, the X-ray spectrum is dominated by bremsstrahlung emission (with a non-negligible contribution from large radii), again up to a maximum energy of $\sim k T_{e}$, beyond which the spectrum falls off exponentially, e.g. [22]. Electron temperatures approaching $10^{10} \mathrm{~K}$ may be achieved for accretion rates much lower than $\dot{m}_{\text {crit }} \sim \alpha^{2}$. In such a case, bremsstrahlung photons can have energies $k T \gtrsim m_{e} c^{2}$ high enough to annihilate, leading to injection of charges on open magnetic field lines. 


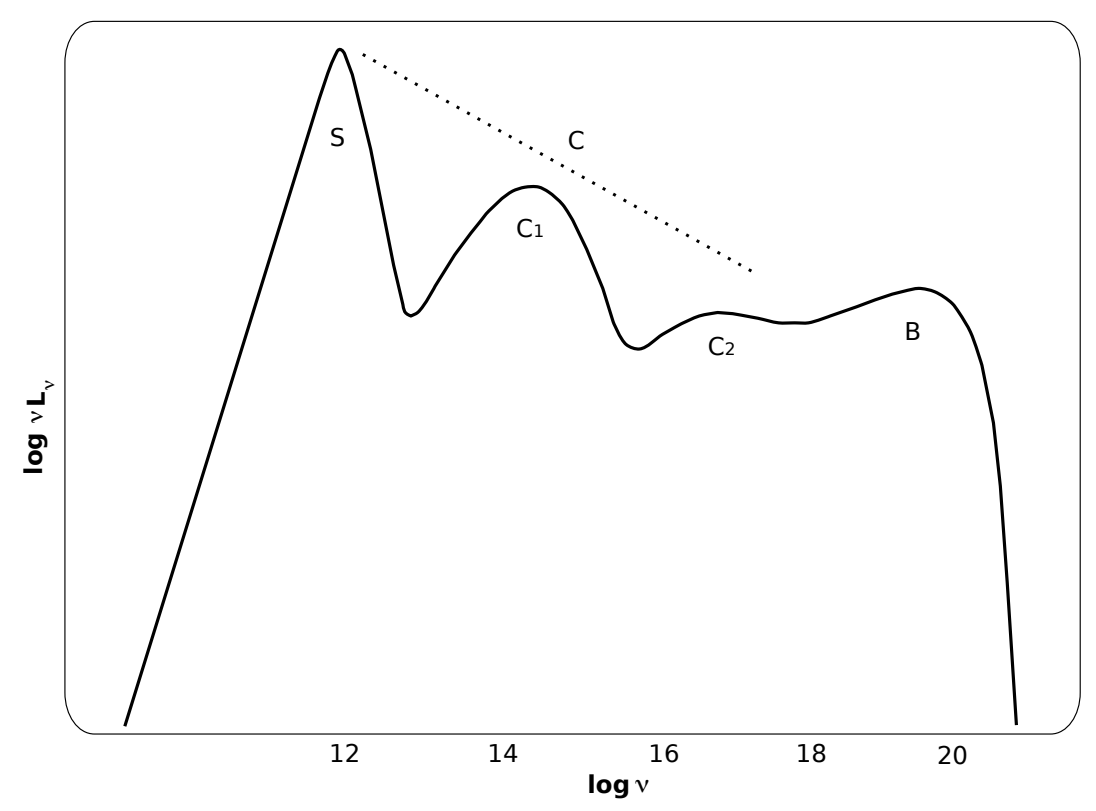

Figure 2: Sketch of a typical ADAF spectrum around a supermassive black hole. Synchrotron emission (S) of the relativistic thermal electrons produces a peak at around $10^{12} \mathrm{~Hz}$. At moderate accretion rates, Comptonization results in a power-law tail (C) extending into the X-ray domain. For low accretion rates, distinct Compton $(\mathrm{C} 1=$ once Compton scattered, $\mathrm{C} 2=$ twice Compton scattered) peaks appear. The $\mathrm{X}$-ray/soft gamma-ray regime is usually dominated by bremsstrahlung emission (B), peaking at $h v \simeq k T_{e}$.

\subsection{Plasma injection into the magnetosphere}

For sufficiently high annihilation rate the charge density injected into the magnetosphere can exceed the Goldreich-Julian (GJ) value everywhere and keep the magnetosphere force-free by screening off the parallel electric field component. However, at lower annihilation rates the magnetosphere will be starved and gaps appear. In that case, the charge density produced by annihilation of $\mathrm{MeV}$ photons from the RIAF defines the multiplicity required to establish a force-free outflow (with charge density $\geq$ GJ density) above the vacuum gap.

The number density of ADAF bremsstrahlung MeV photons is $n_{\gamma} \propto \dot{m}^{2}$ and the production (injection) rate of electron/positron pairs inside the magnetosphere due to $\gamma \gamma$ annihilation is roughly $n_{ \pm} \sim \sigma_{\gamma \gamma} n_{\gamma}^{2} r_{s} \propto \dot{m}^{4}$. In terms of the GJ density $n_{\mathrm{GJ}}=\Omega B /(2 \pi e c) \propto \dot{m}^{1 / 2}$, the ratio becomes [12]

$$
n_{ \pm} / n_{\mathrm{GJ}} \simeq 0.06 \dot{m}_{-4}^{7 / 2} M_{9}^{1 / 2}
$$

As bremsstrahlung depends on $T_{e}$, which itself depends on $\dot{m}$, the numerical estimate for the pair density is somewhat uncertain. The strong dependence on accretion rate suggests, however, that a gap may form during periods of low accretion, so that any emission from the gap may be intermittent. Note that in principle a variety of non-ideal MHD effects may become important when the plasma remains charge-starved, e.g., [23]. 


\section{Particle acceleration and VHE emission in the BH magnetosphere}

A maximally rotating black hole, embedded in an external magnetic field, will generate an electric potential difference across a gap of height $h$, given by

$$
\Delta V_{e}=1.7 \times 10^{20} B_{3} M_{9}\left(h / r_{s}\right)^{2} \quad \text { Volts }
$$

where $B_{3}=B / 10^{3} \mathrm{G}$. Charged particles that get injected into this gap will accelerate and quickly reach a terminal Lorentz factor at which radiative losses balance energy gain, i.e., $q \Delta V_{e}=P t=$ $P h / c$ [2], where $P=P_{\text {cur }}+P_{\mathrm{IC}}$ is the sum of curvature losses,

$$
P_{\text {cur }}=\frac{2}{3} \frac{q^{2} c \gamma^{4}}{\rho^{2}}
$$

with $\rho$ the curvature radius of magnetic field lines, and inverse Compton (IC Thomson) losses

$$
P_{\mathrm{IC}}=\sigma_{T} c \gamma^{2} u_{s}
$$

If the ambient photon field is weak enough (as expected in M87), the energy loss of an electron will be dominated by curvature emission, limiting achievable Lorentz factors in M87 to $\gamma_{c} \sim 3 \times 10^{10}$ [12]. The peak energy of the curvature spectrum is then at a few $\mathrm{TeV}$ at most. However, the maximum energy of IC photons up-scattered by the accelerated electrons can exceed $\gamma_{c} m_{e} c^{2} \simeq 10^{4}$ TeV. At such energies, interactions with the ambient disk radiation field (see Fig. 2) can initiate pair cascades just above the gap, resulting in enough charges to establish a force-free outflow.

Whatever the dominant loss channel (IC or curvature), the maximum $\gamma$-ray power of the gap is determined by the product of charge density times voltage drop, i.e.

$$
L_{\gamma}=\int n_{G J} c\left(e \Delta V_{e}\right) 2 \pi r^{2} d \theta \simeq 3 \times 10^{45} \eta B_{3}^{2} M_{9}^{2}\left(h / r_{s}\right)^{2} \quad \mathrm{erg} \mathrm{s}^{-1},
$$

where $\eta$ is a geometrical factor. For, e.g., $\eta \sim 0.1$ and $\left(h / r_{S}\right)^{2} \sim 10^{-3}$ this output still exceeds the measured TeV luminosity in M87 of several $10^{40} \mathrm{erg} / \mathrm{s}$ [6]. Note that the ratio $L_{\gamma} / L_{B Z}$, where $L_{B Z}$ is the maximum Blandford-Znajek power that can be extracted by a force-free flow, scales as $\left(h / r_{s}\right)^{2}$.

\section{Optical depth for pair production and arising pair multiplicity}

$\mathrm{TeV}$ photons of energy $\varepsilon$ most efficiently interact (producing $e^{+} e^{-}$pairs) with soft photons of energy $\varepsilon_{s} \simeq 1(1 \mathrm{TeV} / \varepsilon) \mathrm{eV}$. In the presence of an ADAF, the relevant soft photon field comes from its synchrotron to Compton branch (see Fig. 2). The optical depth for $\gamma \gamma$-absorption in such an environment shows a sensitive dependence on accretion rate with

$$
\tau \propto \dot{m}^{q}
$$

where $q \geq 2[24,12]$. For M87, $\tau$ is close to unity for photons of a few $\mathrm{TeV}$ and $\dot{m} \sim 10^{-4}$. While moderate changes in accretion rate are therefore expected to make M87 transparent to pair absorption up to photon energies of some $\mathrm{TeV}$, severe absorption seems unavoidable above a few 
tens of $\mathrm{TeV}$ (i.e., for the maximum IC up-scattered photons). The relevant number of photons in the gap (per ADAF-injected electron/positron) at these energies is of order [12]

$$
N_{\gamma}^{\mathrm{IC}} \sim 10^{3} L_{p, 41}\left(h / r_{s}\right)^{3 / 4}
$$

where $L_{p, 41}=L_{p} / 10^{41} \mathrm{erg} / \mathrm{s}$ is the characteristic ADAF synchrotron peak luminosity (that, however, depends again on accretion rate). Equation (2.2) suggests that usually some multiplicity $M>1$ is required to ensure that the magnetosphere is screened out (i.e., $M n_{ \pm} \geq n_{\mathrm{GJ}}$ ). If one takes this multiplicity to be provided by pair production in the gap, i.e., that $N_{\gamma} \geq n_{\mathrm{GJ}} / n_{ \pm}$holds, then Eq. (4.2) implies that a minimum gap size $\left(h / r_{s}\right) \geq 0.01$ is required to ensure a force-free flow. For a fully restored gap, on the other hand, a large multiplicity up to $M \sim 10^{3}$ is expected.

The pair production opacity in the noted ADAF environment decreases with decreasing $\gamma$-ray energy, and becomes sufficiently small below $10 \mathrm{TeV}$ to allow photons at these energies to freely escape the system.

\section{Conclusions}

Recent observations of M87 support a picture where the variable $\mathrm{TeV}$ emission originates in a magnetospheric gap located at the base of the VLBA jet. Injection of charges into such a gap is facilitated by annihilation of $\mathrm{MeV}$ photons from the surrounding ADAF. For the estimated accretion rate in M87, the injected charge density is not sufficient to ensure that the parallel electric field component is screened off. The injected pairs will thus be quickly accelerated in the gap to very high energies, producing curvature VHE photons and up-scattering ambient disk (ADAF) photons to super- $\mathrm{TeV}$ energies. Photons with energies above $10 \mathrm{TeV}$ will be absorbed, producing further pairs and thereby ensuring the formation of a force-free outflow above the gap. We note that rapid variability is naturally accounted for by this mechanism, as even moderate changes in accretion rate will lead to nonlinear fluctuations of the gap potential and the resultant $\mathrm{TeV}$ emission, owing to the sensitive dependence of the density of injected charges on accretion rate.

\section{References}

[1] E. Boldt and P. Ghosh, MNRAS 307 (1999) 491.

[2] A. Levinson, Phys. Rev. Lett. 85 (2000) 912.

[3] Z. Osmanov et al., A\&A 470 (2007) 395.

[4] A. Neronov et al., NJPh 11 (2009) 065015.

[5] F.M. Rieger, IJMPD (2011) submitted.

[6] F. Aharonian et al. (H.E.S.S. Collaboration), Science 314 (2006) 1424.

[7] V.A. Acciari et al. (VERITAS Collaboration), ApJ 679 (2008) 397.

[8] J. Albert et al. (MAGIC Collaboration), ApJ 685 (2008) L23.

[9] V.A. Acciari et al. (VERITAS, MAGIC, VLBA M87 and H.E.S.S. Collab.), Science 325 (2009) 444.

[10] A. Neronov and F.A. Aharonian, ApJ 671 (2007) 85. 
[11] F.M. Rieger and F.A. Aharonian, $A \& A 479$ (2008) L5.

[12] A. Levinson and F.M. Rieger, ApJ 730 (2011) 123.

[13] F. Macchetto et al., ApJ 489579.

[14] K. Gebhardt and J. Thomas, ApJ 700 (2009) 1690.

[15] F.N. Owen, J.A. Eilek and N.E. Kassim, ApJ 543 (2000) 611.

[16] T. Di Matteo et al., ApJ 582 (2003) 133.

[17] C.S. Reynolds et al., MNRAS 283 (1996) L111.

[18] M. Camenzind, in: The radio galaxy Messier 87, ed. H.-J. Röser, \& K. Meisenheimer, LNP 530 (1999), 252.

[19] R. Narayan, in: Lighthouses of the Universe: The Most Luminous Celestial Objects and Their Use for Cosmology, ed. M. Gilfanov et al., Springer (2002), 405.

[20] R. Narayan and I. Yi, ApJ 452 (1995) 710.

[21] R. Mahadevan, ApJ 477 (1997) 585.

[22] T. Manmoto, S. Mineshige and M. Kusunose, ApJ 489 (1997) 791.

[23] J.G. Kirk and I. Mochol, ApJ 709 (2011) 104.

[24] Y.-R. Li et al., ApJ 699 (2009) 513. 